\title{
Gentechnische Experimente zur Beeinflussung der Lignanbiosynthese
}

\author{
E. Fuss \\ Institut für Entwicklungs- und Molekularbiologie der Pflanzen, Pflanzliche Zell- und Gewebekultur, Heinrich-Heine-Universität Düsseldorf \\ Korrespondenz an: E. Fuss, Institut für Entwicklungs- und Molekularbiologie der Pflanzen, Pflanzliche Zell- und Gewebekultur, \\ Heinrich-Heine-Universität Düsseldorf \\ Eingegangen: 5. September 2005
}

Im Gegensatz zum hochmolekularen Lignin sind Lignane Dimere aus 8-8`-verknüpften Phenylpropaneinheiten. Zu den wohl bekanntesten Lignanen zählt das Secoisolariciresinol, dessen Diglucosid in Leinsamen (Linum usitatissimum) zu finden ist. Es besitzt phytoestrogenen Eigenschaften, denen eine Schutzwirkung gegen hormonabhängige Krebsarten zugeschrieben wird. Derivate des in Podophyllum spec. akkumulierten Podophyllotoxins wie Etopophos ${ }^{\circledR}$ werden in der Chemotherapie gegen Krebs eingesetzt. Durch Übersammeln ist die natürliche Quelle für das Podophyllotoxin, Rhizome und Wurzeln von $P$. hexandrum, bedroht. Als Alternative haben wir Zellsuspensions- und „Hairy Root“-Kulturen von L. album etabliert, die Podophyllotoxin bzw. 6-Methoxy-podophyllotoxin akkumulieren. Der Focus unserer Arbeiten liegt in der Aufklärung der Biosynthese des Podopyllotoxins und 6-Methoxypopdo-phyllotoxins in L. album mit dem Ziel, die Ausbeute an Podophyllotoxin durch gentechnische Manipulation zu erhöhen.

Wir haben einige cDNAs für Enzyme des Allgemeinen Phenylpropanstoffwechsels (Phenylalanin-Ammoniak-Lyase, Zimtsäure-4-Hydroxylase, Zimtalkohol-Dehydrogenase, Cinnamoyl-CoA-Reduktase) isoliert. Daneben klonierten wir eine cDNA für die Pinoresinol-Lariciresinol-Reduktase, welche an den frühen spezifischen Schritten der Liganbiosynthese beteiligt ist. Ein Transformationsprotokoll zur Überexpression bzw. zu RNAi-Experimenten wurde zunächst für L. album entwickelt und jetzt auch auf andere Leinarten übertragen. Dabei werden für die Transformation mit Agrobacterium tumefaciens entwickelte Vektoren zur Übertragung der Genkonstrukte ins Pflanzengenom mittels A. rhizogenes verwendet. Als Selektionsagenz wird Paromomycin eingesetzt, da Hairy Roots der bisher untersuchten Leinarten auch ohne Resistenzgen in Medien mit bis zu 300 mg/l Kanamycin wachsen. Die Überexpression der Pinoresinol-Lariciresinol-Reduktase in Hairy Roots von $L$. album führte wahrscheinlich zu einem Cosuppressionseffekt. Hairy Roots mit Überexpressionskonstrukt zeigten einen geringeren Gehalt an 6-Methoxy-podophyllotoxin als die Kontrollinien. RNAi-Experimente zur Minimierung der Expression der Pinoresinol-Lariciresinol-Reduktase in L. album, L. perenne und L. strictum ssp. corymbulosum, die Lignane verschiedener Subtypen akkumulieren, werden gerade unternommen. Diese Experimente sollen zeigen, ob eine Pinoresinol-LariciresinolReduktase an der Biosynthese der verschiedenen Lignane beteiligt ist.

Der DFG und dem BMBF wird für ihre finanzielle Unterstützung gedankt. 